\title{
Photovoltaic Cells with Increased Voltage Output for Optical Power Supply of Sensor Electronics
}

\author{
Henning Helmers, Lukas Wagner, César E. Garza, S. Kasimir Reichmuth, Eduard Oliva, \\ Simon P. Philipps, David Lackner, Andreas W. Bett \\ Fraunhofer Institute for Solar Energy Systems ISE, Heidenhofstraße 2, 79110 Freiburg, Germany, \\ henning.helmers@ise.fraunhofer.de
}

\begin{abstract}
An elegant solution for the power supply of sensor electronics is the application of power-by-light technology. With this technology several challenges related to conventional copper wiring are inherently overcome; benefits are galvanic isolation, the suppression of electromagnetic interference, and the possibility to combine power and bidirectional data transmission in a single fiber link.

In order to power sensor electronics efficiently, a supply voltage in the range 3 to $12 \mathrm{~V}$ is typically required. This paper deals with the photovoltaic laser power converter used to convert the transmitted optical power back into electricity. The advanced cell concepts of multi-junction and multi-segment cells are discussed which both aim at an increased output voltage on the device level, thereby eliminating the need for additional DC/DC conversion. Modeling and experimental results of GaAs based cells are presented, namely of single-junction 2- to 12-segment cells as well as of a dualjunction single-segment structure. A discussion of the pros and cons of the different concepts is given, focusing on the consequences of misalignment and temperature changes.
\end{abstract}

Key words: Laser power converter, misalignment, multi-junction, multi-segment, photovoltaic cell, power by light, temperature, voltage.

\section{Introduction}

One important aspect in sensor design is the power supply. Besides conventional copper wiring, an elegant solution is to power the sensor electronics optically. This can be realized by optical power transmission of laser light from the base station to a photovoltaic receiver located at the sensor which converts the monochromatic laser light back into electricity.

With such power-by-light technology several challenges related to conventional copper wiring are inherently overcome, such as electromagnetic interference, the risk of short circuits and sparks, or susceptibility to corrosion. Furthermore, it provides galvanic isolation, lightning protection, weight reduction, the possibility of wireless powering, and the compatibility with rotating systems. An additional benefit is the possibility to combine power and bidirectional data transmission into a single fiber link. Fields of application are manifold: Examples are structural health monitoring of wind turbines [1,2], fuel gauges in aircraft wings [3], monitoring of high voltage power lines $[4,5]$, optical powering of automotive sensors [6], biosensors in smart implants [7-9], monitoring of passive optical networks [10], and fully optical sensor networks $[11,12]$.

In order to power sensor electronics efficiently, a supply voltage in the range 3 to $12 \mathrm{~V}$ is typically required. This paper deals with photovoltaic laser power converters used in power-by-light technology. Two advanced cell concepts, namely multi-junction and multisegment cells, are discussed which aim at an increased output voltage on the device level, thereby eliminating the need for additional DC/DC conversion. Experimental and modeling results of GaAs based cells are presented, namely of single-junction 2- to 12-segment cells as well as of a dual-junction single-segment cell. The paper concludes with a discussion of pros and cons of these concepts, focusing on the consequences of misalignment and temperature changes.

\section{Photovoltaic Laser Power Converters}

With photovoltaic cells as power converters for monochromatic light, very high opto-electrical conversion efficiencies can be achieved because the semiconductor material's bandgap 
can be well matched to the energy of the photons. With III-V compound semiconductors, a broad range of bandgap energies from below 0.3 to $2.5 \mathrm{eV}$ is covered, corresponding to laser wavelengths in the range 0.5 to $4 \mu \mathrm{m}$. With an adjusted design, thermalization and transmission losses, as the main loss mechanisms in solar cells, can be minimized due to the matching of bandgap and photon energy. A plot of the monochromatic efficiency of an idealized laser power converter is shown in Fig. 1 as a function of laser wavelength for different irradiances. The curves are calculated in the detailed balance limit [13] under the assumptions of monochromatic irradiance, a semiconductor bandgap $E_{g}$ that matches the laser wavelength $\lambda\left(E_{g}=h c / \lambda\right)$, full absorption of the incident photons, each absorbed photon contributes one charge carrier to the photo current (IQE=EQE=1), and radiative recombination only [14]. As can be seen in the plot, the ideal conversion efficiency in the detailed balance limit evaluates to values above $80 \%$ for an irradiance of $100 \mathrm{~W} / \mathrm{cm}^{2}$. It is remarked that for practical devices somewhat lower values are expected. However, a comparison with the detailed balance limit for an ideal single-junction solar cell under one sun illumination, which is $33 \%$ [15], reveals the possibilities of monochromatic light conversion.

A semiconductor material that is frequently used for laser power converters is GaAs. With a bandgap of $1.42 \mathrm{eV}$ it is well matched for the $800-850 \mathrm{~nm}$ wavelength range. Due to its vast use in micro- and optoelectronics, it is a wellknown material and available in excellent quality. At Fraunhofer ISE, a conversion efficiency of $57.4 \%$ was achieved with a GaAs based photovoltaic laser power converter under $805 \mathrm{~nm}$ laser light at an irradiance of 124.0 W/cm². For comparison, the highest reported opto-electrical conversion efficiency of a solar cell is $46 \%$ under concentrated light [ 16 , 17].

A drawback of photovoltaic cells, however, is the output voltage of a single cell. For a GaAs based photovoltaic cell it is about $1 \mathrm{~V}$. In contrast, to power sensor electronics efficiently, supply voltages in the range 3 to $12 \mathrm{~V}$ are required. To overcome this limitation, photovoltaic laser power converter devices with an increased voltage output were developed. An increased output voltage can be achieved by connecting several subcells in series. That way the output voltage of the string corresponds to a multiplication of the single cell voltage with the number of subcells. A monolithic series connection can be

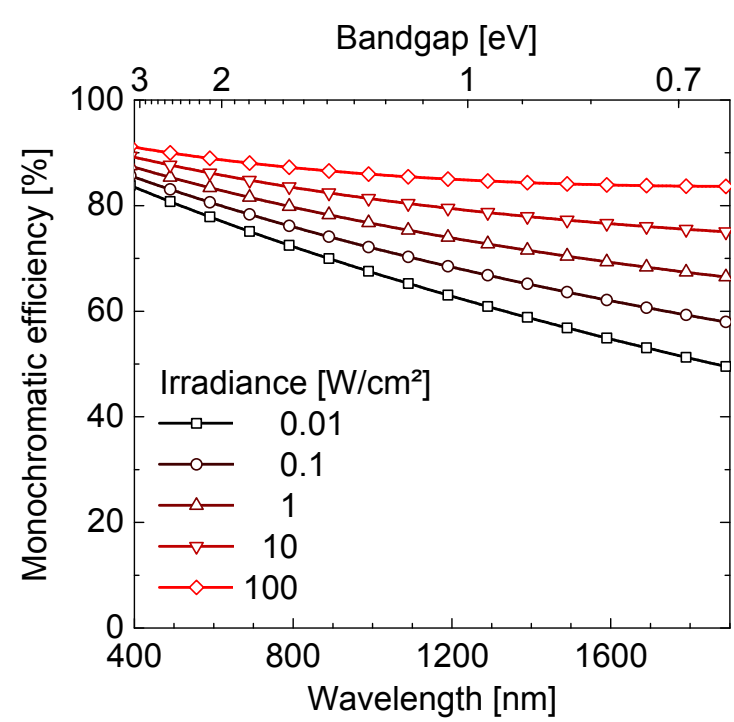

Fig. 1. Detailed balance calculation of the monochromatic efficiency of ideal laser power converters in the radiative limit. Assumptions: Monochromatic irradiance, $\mathrm{E}_{\mathrm{g}}=\mathrm{hc} / \lambda$, full absorption of the incident photons, each absorbed photon contributes one charge carrier to the photo current $(\mathrm{IQE}=\mathrm{EQE}=1)$, dark current determined by radiative recombination only. After Ref. [14].

implemented by vertical stacking of subcells (multi-junction cells) or by lateral segmentation (multi-segment cells, also known as monolithic interconnected modules, MIMs) [18]. In the following section these two concepts are introduced.

\section{Cell Concepts for Increased Voltage Output}

To realize vertically interconnected stacks, the subcells are grown monolithically on top of each other on a conductive substrate [18]. Fig. 2 shows a cross section of a dual-junction structure as an example. In between each pair of subcells a tunnel junction is implemented to establish a low-loss series connection. The contacts to the device are established by metallization of front and back side of the device. To improve lateral conduction to the front grid fingers, a transparent lateral conduction layer can be implemented above the top cell $[18,19]$. Due to the growth of several subcells of different thicknesses and the necessity of tunnel diodes in between, the epitaxial growth of multi-junction cells is rather complex. The manufacturing, however, is similar to single-junction cell processing.

To realize multi-segment cells or monolithic interconnected modules (MIMs) [20], the photovoltaic cell structure is grown on semiinsulating substrate. Fig. 3 illustrates the interconnection scheme. The electrical separation of the individual subcells is created by etching isolation trenches through the 


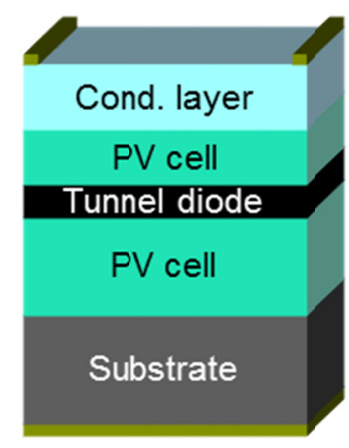

Fig. 2. Cross section of a symmetry element of a stacked dual-junction structure. The two subcells are interconnected via a tunnel diode. An upper conduction layer improves the lateral conduction to the front grid metallization.

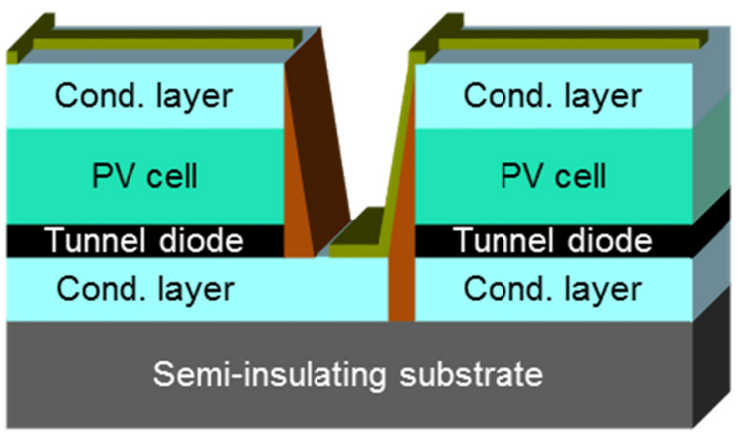

Fig. 3. Schematic illustration of the interconnection scheme of monolithic interconnected modules to realize multi-segment cells.

structure into the semi-insulating (nonconductive) substrate $[18,21]$. Below the photovoltaic cell, a highly conductive lateral conduction layer is implemented and connected with a tunnel diode. To contact the back side of the subcells this layer is partially exposed during manufacturing. The flanks of the etched trenches are protected using a dielectric material. The series connection is finally realized via a front metallization which interconnects the lower conduction layer as the back contact of one subcell with the front grid of the adjacent subcell. Similar to multi-junction cells a transparent lateral conduction layer above the photovoltaic cell improves conduction to the front contact. In total, the epitaxial structure for multi-segment cells is of moderate complexity. However, the manufacturing is rather complex: At least six photolithography steps are required which is twice as much as is required for conventional single-segment processing.

Due to the series connection, in both concepts the subcell that generates the lowest photo current limits the current of the full device. For that reason, the devices must be carefully designed to achieve equal current generation in each subcell. For multi-junction cells, currentmatching is achieved by precisely tuning the thicknesses of the stacked subcells in a way, that each subcell absorbs the same fraction of the incident monochromatic light. Due to BeerLambert law of exponential absorption this means that the cell thickness decreases from bottom to top cell, and the upmost cell thickness decreases with increasing number of junctions. For multi-segment cells, current matching is achieved by proper design of the geometry of the subcell segments with respect to the profile of the light spot. Since the light spot usually features circular symmetry, a pieshaped design is common.

\section{Investigated Test Cells}

Single-junction multi-segment cells based on GaAs were manufactured in different designs. The realized designs differ in the number of segments, the size, and the front grid metallization. Regarding segmentation, multisegment cells with $N=2,4,6$, and 12 segments were realized. For all $N$, cells with a radius of $1.04 \mathrm{~mm}$ were realized; in addition, the 2, 4, and 6 segment designs were also realized with smaller radii of $0.75 \mathrm{~mm}$ and $0.5 \mathrm{~mm}$. Fig. 4 shows microscope pictures of example cell designs in different configurations: (a) small 2segment cell, (b) medium 4-segment cell, (c) large 6-segment cell, (d) large 12-segment cell. Each design was further realized with varying front grid designs to optimize for shading and series resistance losses. It is noted that the designs shown in Fig. 4 feature no front grid to minimize shading loss.

As a test structure for the vertically stacked multi-junction approach, dual-junction GaAs/ GaAs cells in single-segment design were investigated.

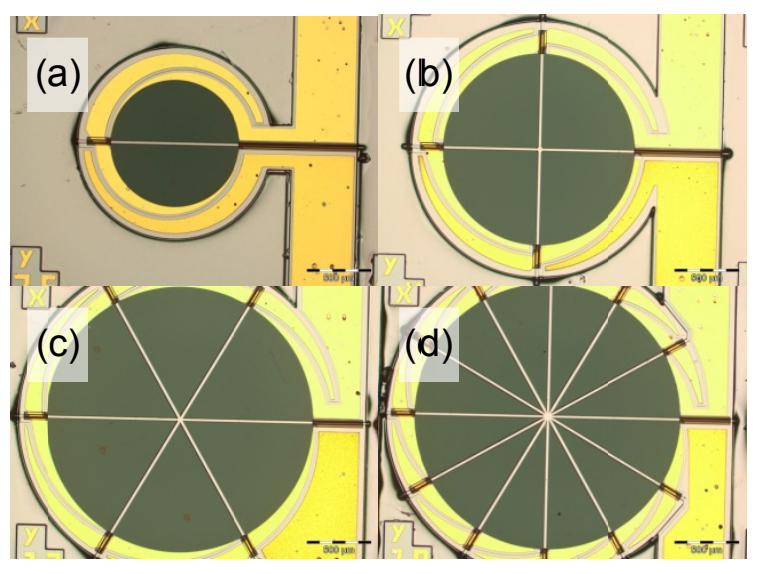

Fig. 4. Microscope pictures of experimentally realized multi-segment designs. Designs with 2, 4, 6, and 12 segments were realized in different sizes with radii of the active area of $0.5,0.75$, and $1.04 \mathrm{~mm}$. All designs feature large metal pads for easy contacting of the front (top right) and back (bottom right) contacts at the right side of the chip. 


\section{Increased Output Voltage}

Investigation of the open circuit voltages $V_{O C}$ yields insight into the functionality of the interconnection. Fig. 5 shows measured open circuit voltages $V_{O C}$ as a function of short circuit current density $J_{S C}$ for the single-junction multisegment designs (half-filled symbols) and the dual-junction single-segment cell (crosses). All designs are functional and their output voltages multiply with the number of subcells. It is notable that the open circuit voltages of the 1-junction 2-segment and the 2-junction 1-segment design are nearly the same, as also reported by Schubert et al. [18]. It is remarked that the voltage at maximum power point $V_{m p}$ is a priori lower than the open circuit voltage $V_{O C}$. The former can be significantly reduced due to the series resistance of the specific cell design (mainly determined by front grid metallization and size) as well as by current mismatching of individual subcells. An evaluation and assessment of how segmentation, size and grid design influence the performance of the cells will be published elsewhere.

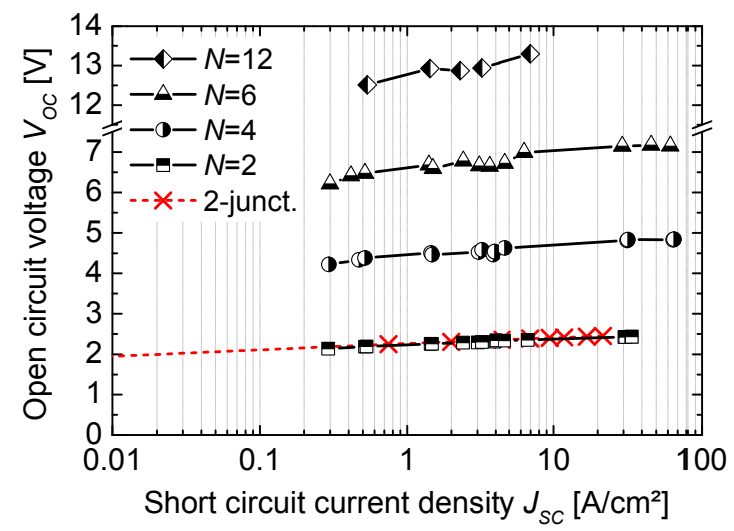

Fig. 5. $\mathrm{V}_{\mathrm{OC}}-\mathrm{J}_{\mathrm{SC}}$ pairs of different single-junction multi-segment cells $(\mathrm{N}=2,4,6,12)$ and a dualjunction single-segment cell measured under variable irradiance. The multiplication of $\mathrm{V}_{\mathrm{OC}}$ by the number of subcells can be clearly observed.

\section{Misalignment Tolerance}

When laser power converters are implemented into an actual system, an important issue is the influence of misalignment of the light spot on the performance. From a manufacturing point of view this goes along with the question of how accurate the placing, fiber coupling, etc. needs to be, or in other words which manufacturing tolerances are acceptable.

For multi-junction single-segment cells the misalignment tolerance is similar to the one of single-junction single-segment cells. For pieshaped multi-segment cells, however, the sensitivity to misalignment becomes more pronounced as the number of segments increases. The reason for this behavior is that misalignment results in significant current mismatch between the subcells. In the following, the results of a recent study by Wagner et al. are summarized where a generally applicable modeling approach for quantification of this behavior is presented [22]. Fig. 6 illustrates how misalignment affects a 4-segment cell. Assuming a homogeneous light spot of equal size as the photovoltaic cell, an increasing displacement between cell and light spot leads to a significant reduction of the incident optical power on the segment which is located on the opposite side compared to the direction of displacement. Thus, this segment generates a reduced photo current and, consequently, limits the overall current. When the displacement equals the cell radius, the overlap of light spot and limiting subcell vanishes and the current drops to zero. For a quantitative assessment the parameter maximum acceptable misalignment $M A M_{90}$ is introduced. It is defined as the displacement which results in a current drop to $90 \%$ of the ideal current. For a circular single-segment cell without segmentation it equals $15.7 \%$ of the cell radius $r$ [22]. In general, the acceptable misalignment is direction dependent. For symmetry reasons there exists a best and a worst case axis, where for pie-shaped cells the best case is along the separation trench and the worst case is along the center of a segment. With increasing number of segments $N$ the maximum acceptable misalignment quickly drops towards a limit value of $5.1 \%$ of $r$ [22], i.e. $53 \mu \mathrm{m}$ for the large cells presented here. It is remarked that with respect to the actual system requirements the maximum acceptable misalignment $M A M_{x}$ can also be defined using a different percentage value $x$.

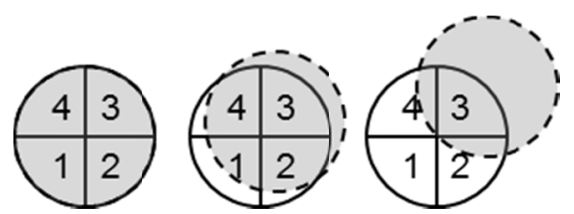

Fig. 6. Illustration of a multi-segment cell with subcells 1 to 4, illuminated with an equally sized, homogeneous light spot (dashed circle). Increasing misalignment in direction of the center of segment 3 significantly reduces the overllap of the light spot with segment 1 and, thereby, causes a significant current mismatch. After Ref. [22].

\section{Temperature Dependence}

For laser power converters the operating temperature can span a broad range. The actual temperature of the laser power converter influences its performance. One important effect is the shift of the bandgap $E_{g}$ of the material [23]. To ensure proper absorption of 


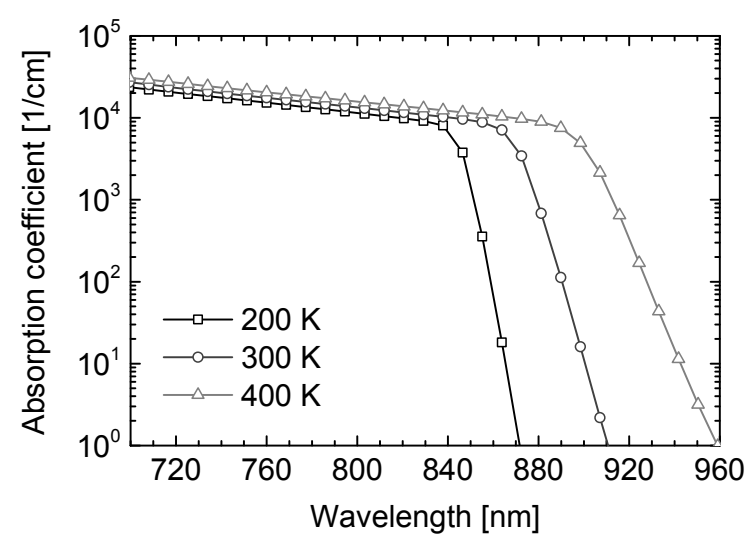

Fig. 7. Plot of the absorption coefficient of $\mathrm{GaAs}$ as a function of wavelength. Data from Ref. [24].

the incident laser light, the cutoff wavelength, $\lambda_{c}=h c / E_{g}$, must be kept above the laser wavelength. Otherwise, the semiconductor becomes transparent for the laser light and the performance breaks down immediately. Moreover, the temperature affects the absorption coefficient also for wavelengths below the cutoff wavelength, namely it increases with increasing temperature. Fig. 7 shows the temperature dependence of the absorption coefficient of GaAs from $200 \mathrm{~K}$ to $400 \mathrm{~K}$ based on data published by Philipps et al. [24]. In principle, lower absorption leads to a reduced photo current. However, the effect is small: Under the assumption of an internal quantum efficiency of 1 , the data shown in Fig. 7 translates into a temperature coefficient of the current of only $0.01 \% / \mathrm{K}$. The temperature coefficient of the open circuit voltage is determined by the material; for GaAs it is known to be in the range $-0.26 \% / \mathrm{K}$ to $-0.11 \% / \mathrm{K}$, where lower absolute values are reached at higher irradiances [25, 26].

For laterally segmented cells, temperature affects all segments equally. Thus, the current matching of single-junction multi-segment cells is independent of temperature.

For multi-junction cells, however, the situation is different. The subcell thicknesses are designed in a way that all subcells absorb an equal fraction of the incident monochromatic light. For that reason, the layer design is optimized for a given wavelength and temperature. Deviations from the design case lead to current mismatch between the subcells and the overall current becomes limited by the subcell that absorbs the least fraction of the incident light.

Based on the data shown in Fig. 7 and assuming an internal quantum efficiency of 1 , the related temperature coefficient of the overall current is calculated for multi-junction cells with up to 12 subcells designed for $300 \mathrm{~K}$ and

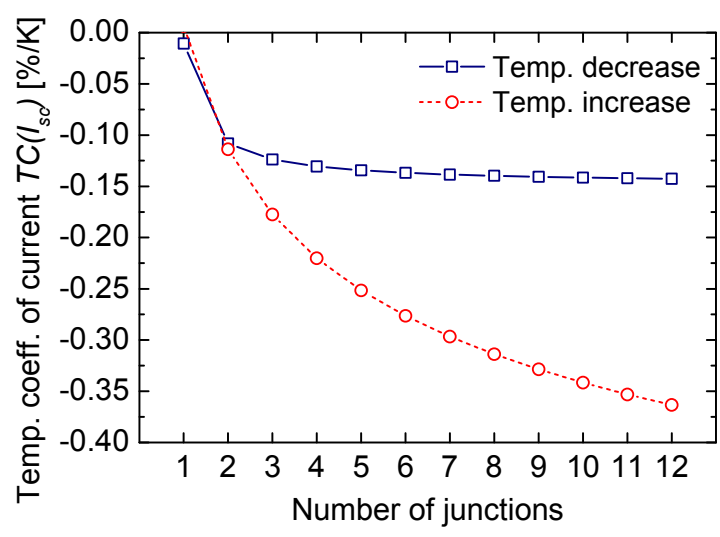

Fig. 8. Plot of the temperature coefficient of the current related to temperature induced current mismatch in multi-junction laser power converters. The values are calculated for a laser wavelength of $810 \mathrm{~nm}$ and a bottom cell thickness of $3.6 \mu \mathrm{m}$.

$810 \mathrm{~nm}$ laser light but operated at respectively lower and higher temperatures. The results are shown in Fig. 8, assuming a bottom cell thickness of $3.6 \mu \mathrm{m}$. The negative values are to be understood as a drop in the overall mismatched current compared to the ideal current matched case at $300 \mathrm{~K}$. It is striking that for the dual-junction case increasing and decreasing temperature lead to a similar mismatch of about $-0.11 \% / \mathrm{K}$. For three and more junctions, however, a temperature increase (circles) affects the current matching much worse than a temperature drop (squares). This behavior can be understood from the following considerations: Temperatures above the design temperature result in an increased absorption in the top cell and, consequently, less light is transmitted into the underlying layers. Consequently, for higher temperatures always the undermost subcell limits the device current. For lower temperatures the opposite is true. Increasing the number of junctions at low temperatures means that the current is limited by the upmost cell (of decreasing thickness). On the other hand, for high temperatures the incident light that reaches the limiting bottom cell is filtered by an increasing number of above lying subcells with a disproportional total thickness increase.

Finally, it is remarked that the current mismatch in multi-junction laser power converters can be counterbalanced by an effect known as luminescence coupling [27]. If carriers which are generated in the overproducing subcells recombine radiatively, they emit a photon which can be reabsorbed by adjacent subcells. As a result, the incident photons are redistributed within the stack in a self-organized fashion. Yet, this effect requires radiative recombination to be the dominant process, which requires an excellent material quality. 
Table 1: Comparison of pros and cons of singlejunction single-segment (standard) cells with multijunction and multi-segment laser power converters.

\begin{tabular}{|c|c|c|c|}
\hline & Standard & $\begin{array}{c}\text { Multi- } \\
\text { junction }\end{array}$ & $\begin{array}{c}\text { Multi- } \\
\text { segment }\end{array}$ \\
\hline Output voltage & $\sim 1 \mathrm{~V}$ & $\sim N * 1 \mathrm{~V}$ & $\sim N * 1 \mathrm{~V}$ \\
\hline $\begin{array}{r}\text { Max. acceptable } \\
\text { misalignment }\end{array}$ & $r^{*} 16 \%$ & $r * 16 \%$ & $r^{*} 5-8 \%$ \\
\hline $\begin{array}{l}\text { Temperature } \\
\text { dependence }\end{array}$ & (:) & $\begin{array}{c}: \\
\text { (incr. with } N \text { ) }\end{array}$ & ;:) \\
\hline Epitaxy & Simple & Complex & Moderate \\
\hline $\begin{array}{r}\text { Manufacturing } \\
\text { (photolithography } \\
\text { steps) }\end{array}$ & $\underset{(3)}{\text { Simple }}$ & $\underset{(3)}{\text { Simple }}$ & $\underset{(\geq 6)}{\text { Complex }}$ \\
\hline
\end{tabular}

\section{Summary and Conclusion}

The concepts of multi-junction and multisegment laser power converters were presented. GaAs based single-junction multisegment cells with $2,4,6$, and 12 segments as well as dual-junction single-segment cells were demonstrated. By investigating the open circuit voltage it was shown that both cell concepts are well capable of multiplying the output voltage on the device level by the number of subcells.

Misalignment and temperature changes were discussed in light of their influence on the performance and current matching of theses cell concepts. Multi-segment cells are prone to misalignment, where the sensitivity increases with the number of segments. However, temperature changes do not harm the current matching. For multi-junction cells the situation is the opposite: The influence of misalignment is similar to that of single-junction single-segment cells, whereas temperature changes can cause significant current mismatch between the subcells. To assist optimal system design, the pros and cons of the different cell concepts, as discussed in this paper, are summarized in Table 1.

\section{References}

[1] Frankenstein, Fischer, Weihnacht, Rieske, Proc. 6th EWSHM, (2012).

[2] Worms, Klamouris, Wegh, Meder, Volkmer, Philipps, Reichmuth, Helmers, Bett, Vourvoulakis, Koos, Freude, Leuthold, Stork, Proc. 17th ITG/GMASymposium: Sensors and Measuring Systems, Nürnberg, Germany, 1-4 (2014).

[3] Basanskaya, IEEE Spectrum (2005).

[4] Andersson, Destefan, Ramboz, Weiss, DeHaan, Proc. IEEE/PES TDC, 379-84 (2001); doi: 10.1109/tdc.2001.971264

[5] de Nazaré, Werneck, Proc. IEEE/PES TDC, 1-6 2010); doi: 10.1109/tdc.2010.5484461
[6] EPIC Report, Photonic Technologies for the Automotive Industry, p. 28 (2014).

[7] Murakawa, Kobayashi, Nakamura, Kawata, IEEE Engineering in Medicine and Biology Magazine 18(6), 70-2 (1999); doi: 10.1109/51.805148

[8] Goto, Nakagawa, Nakamura, Kawata, IEEE Transactions on Biomedical Engineering 48(7), 830-3 (2001); doi: 10.1109/10.930908

[9] Algora, Peña, Artificial Organs 33(10), 855-60 (2009); doi: 10.1111/j.1525-1594.2009.00803.x

[10] Roeger, Hiba, Hoh, Hehmann, Pfeiffer, Huebner, Becker, Leuthold, Freude, Proc. NFOEC, (2010); doi: 10.1364/nfoec.2010.nwc4

[11] Roeger, Boettger, Dreschmann, Klamouris, Huebner, Bett, Becker, Freude, Leuthold, Optics Express 16(26), 21821-34 (2008); doi:

10.1364/oe.16.021821

[12] Budelmann, Krieg-Brückner, Journal of Intelligent Material Systems and Structures 24(18), 2183-8 (2013); doi: 10.1177/1045389x12462647

[13] Shockley, Queisser, Journal of Applied Physics 32(3), 510-9 (1961).

[14] Bett, Dimroth, Lockenhoff, Oliva, Schubert,

Proc. 33rd IEEE PVSC, 1-5 (2008); doi:

10.1109/pvsc.2008.4922910

[15] Martí, Araújo, Solar Energy Materials and Solar Cells 43(2), 203-22 (1996); doi: 10.1016/0927-

0248(96)00015-3

[16] Tibbits, Beutel, Oliva, Karcher, Siefer, Dimroth, Bett, Krause, Piccin, Blanc, Munoz-Rico, Arena, Guiot, Charles-Alfred, Drazek, Wekkeli, Farrugia, Hoarau, Schachtner, Tauzin, Signamarcheix, Hannappel, Schwarzburg, Dobrich, Grave, Janin, Wasselin, Proc. 29th EU PVSEC, 1975-8 (2014); doi: 10.4229/eupvsec20142014-4cp.2.1

[17] Fraunhofer ISE, Press Release: New World Record for Solar Cell Efficiency at 46\% (01.12.2014). [18] Schubert, Oliva, Dimroth, Guter, Löckenhoff, Bett, IEEE Transactions on Electron Devices 56(2), 170-5 (2009); doi: 10.1109/ted.2008.2010603

[19] Oliva, Dimroth, Bett, Progress in Photovoltaics:

Research and Applications 4(16), 289-95 (2008); doi: 10.1002/pip.811

[20] Borden, Applied Physics Letters 35(7), 553-4 (1979); doi: 10.1063/1.91176

[21] Helmers, Oliva, Bronner, Dimroth, Bett, IEEE Transactions on Electron Devices 57(12), 3355-60 (2010); doi: 10.1109/ted.2010.2076190

[22] Wagner, Bett, Helmers, On the Misalignment Tolerance of Photovoltaic Laser Power Converters, submitted for publication.

[23] Varshni, Physica 34(1), 149-54 (1967); doi: 10.1016/0031-8914(67)90062-6

[24] Philipps, Stetter, Hoheisel, Hermle, Dimroth, Bett, Proc. 23rd EU PVSEC, 114-7 (2008); doi: 10.4229/23rdeupvsec2008-1bo.4.4

[25] Siefer, Abbott, Baur, Schlegl, Bett, Proc. 20th EU PVSEC, Barcelona, Spain, 495-8 (2005).

[26] Helmers, Schachtner, Bett, Solar Energy Materials and Solar Cells 116, 144-52 (2013); doi: 10.1016/j.solmat.2013.03.039

[27] Walker, Höhn, Micha, Wagner, Helmers, Bett, Dimroth, Impact of Photon Recycling and Luminescence Coupling on III-V Photovoltaic Devices, Journal of Photonics for Energy, in press (2015). 